\section{OPTICAL FIBRE DIAMETER VARIATIONS AND THEIR EFFECT ON BACKSCATTER LOSS MEASUREMENTS}

Indexing terms: Optical fibres, Time-domain reflectometry

Diameter variations in optical fibres are shown to affect backDiamet loss measurements by a mechanism which primarily scatter loss measurements by a mechanism which privelling light. A simple model which predicts anticorrelated loss features when measured from opposite ends of the fibre is verified experimentally.

Introduction: In a recent publication ${ }^{1}$ it was shown that the commonly observed features in fibre local attenuation plots obtained by the backscattering method are associated with fibre diameter fluctuations. In particular, propagation through a contracting taper produces a reduction in observed attenua tion, whereas an expanding taper exhibits a positive loss of similar magnitude. Both effects are the opposite of that which might be intuitively expected. We show here that the phenomenon is caused by attenuation of the backscattered light which, since it contains high order modes, is sensitive to mode conversion and differential mode attenuation (DMA).

Theory: The backscattered power $P_{R}$ received by the detector is affected by both the forward loss of the probe pulse and the reverse attenuation of the scattered light. For simplicity, we assume that the fibre baseline loss $\alpha$ is equal in both directions and thus the returned power $P_{R}=P_{0} e^{-2 x l}$, where $l$ is the distance to the scatter point and $P_{0}$ the received power from the start of the fibre. Further, we assume that the forward propagating pulse only partially excites the guide so that it is able to negotiate small diameter changes without loss.

(i) Expanding taper: The light returned to the detector from a scatter point within or beyond the taper region suffers an additional loss as the high order modes of the larger waveguide, which have been excited by the scattering process, are lost by radiation [Fig. 1(i)]. If we assume that scattering excites all reverse-propagating modes equally, the magnitude of the loss is given simply by the reduction in area of the guide, i.e.

$$
P_{k}=P_{0} e^{-2 n i}\left[d_{0}^{2} / d^{2}(l)\right]
$$
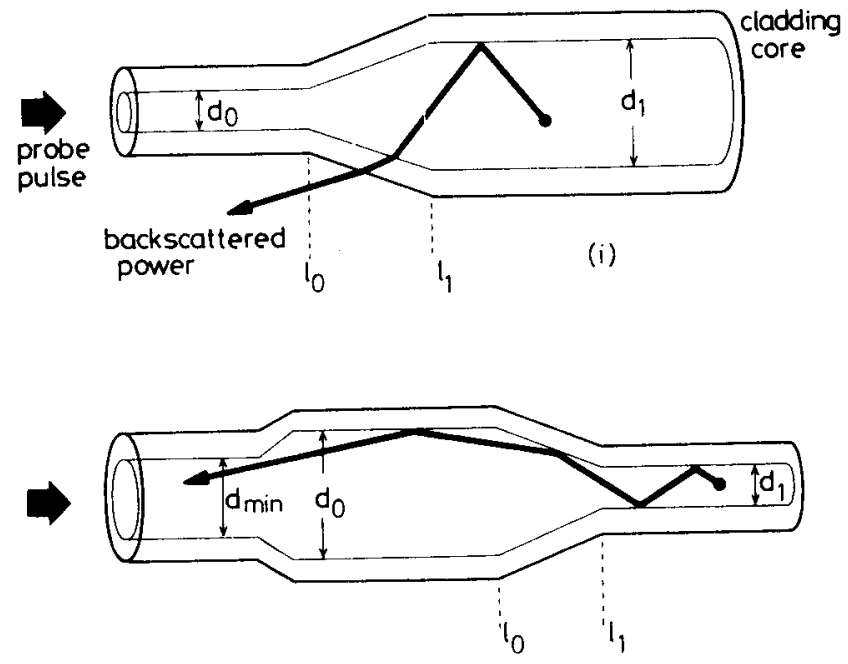

(ii)

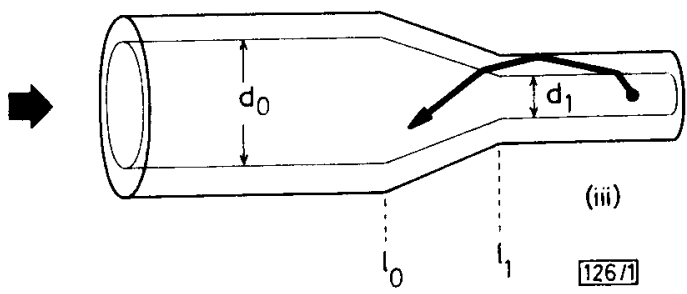

Fig. 1 Schematic representation of effect of fibre tapers on backscattered light where $d(l)=d_{0}$ for $l<l_{0}, d(l)=d_{1}$ for $l>l_{1}$ and $d(l)$ varies linearly for $l_{0}<l<l_{1}$

(ii) Contracting taper with DMA: The situation for propagation into a contracting taper [Fig. 1(ii)] is more complex. An obvious mechanism ${ }^{1}$ which leads to an increased power level being received at the source for scatter points following the taper involves mode conversion, followed by DMA. Light scattered into high order modes from a point after the taper is converted to lower order modes on traversing the taper, since it appears to be an expanding taper to reverse-propagating light. Thus light from beyond the taper returns to the source as lower order modes, whereas that from a point prior to the taper fully excites the guide. Since the fibre has a lower loss when partially excited, a greater proportion of light from beyond the taper arrives at the source than from before the taper, giving an apparent reduction in loss. Note that the cause of the DMA may be intrinsic to the fibre, mode selection at the optical receiver, or a reduction in fibre diameter between the point of interest and the detector. In the latter case, the effect of the contracting taper is to recoup returning power which, for points prior to the taper, had previously been lost at the diameter reduction.

If the DMA is introduced by a prior diameter reduction with minimum diameter $d_{\min }$ [Fig. 1(ii)], and assuming that the scattering process excites all modes equally, then the received power on traversing a contracting taper will increase in proportion to the area change.

$$
\begin{aligned}
P_{K} & =P_{0} e^{-2 \times l}\left[d_{0}^{2} / d(l)^{2}\right] & & \text { for } d(l)>d_{\min } \\
& =P_{0} e^{-2 \times l}\left(d_{0}^{2} / d_{\min }^{2}\right) & & \text { for } d(l) \leq d_{\min }
\end{aligned}
$$

(iii) Contracting taper with cladding effect: Experiments to confirm the above mechanism have shown that a similar increase in backscattered power is apparent on traversing a contracting feature, even when no diameter reduction is present between the source and taper. A second mechanism therefore exists which we explain as follows [Fig. 1(iii)]. A scatter point following the taper excites both the fibre core and cladding and backscattered light propagates in the two regions, particularly if the cladding has low loss (e.g. is silicone coated). When the cladding light encounters the taper, which now appears expanding, it is able to enter the core. By reciprocity with case

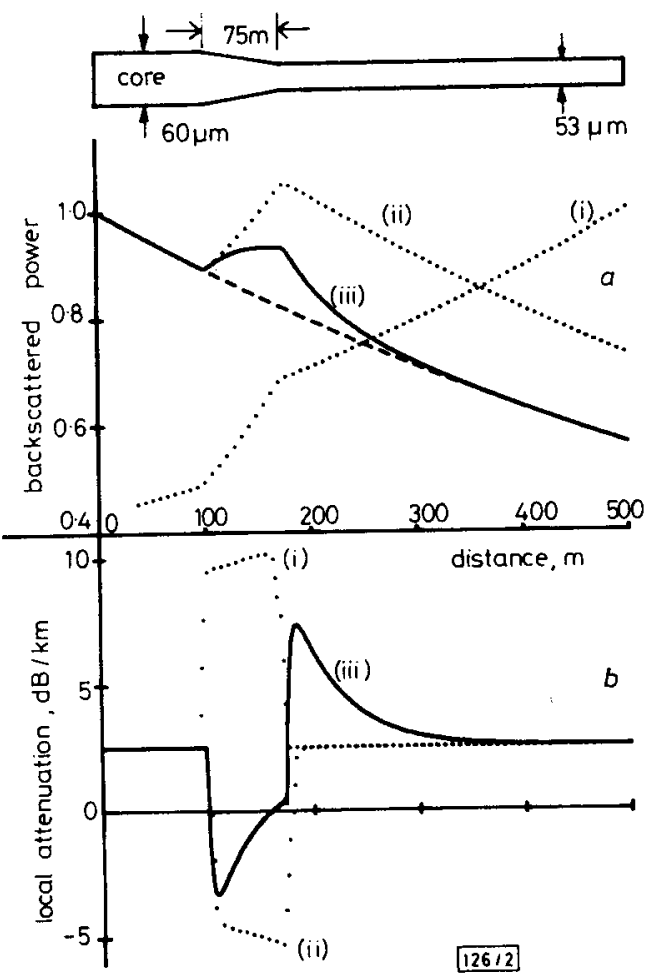

Fig. 2 Backscattered power and local attenuation calculated for mechanisms (i) to (iii) (see text) with core and cladding losses of 2.5 and 100 $\mathrm{d} B / \mathrm{km}$ respectively. Taper dimensions are given schematically 
(i) above, the power which can enter the core is proportional to the area change. Thus

$$
P_{R}=P_{0} e^{-2 x l}\left(d_{0}^{2} / d(l)^{2}\right)
$$

There is now no requirement for DMA in the reverse path and the limitation on the increase in power level given by eqn. 3 is no longer present. Note that the magnitude of the effect is identical to that of eqn. 2 and exactly reciprocal to that obtained for pulse propagation in the reverse direction (eqn. 1).

In practice, cladding modes suffer a higher loss than power in the core, and this provides a means whereby the two power increasing mechanisms can be distinguished. Fig. $2 a$ shows backscattered power calculated for cases (i) to (iii) above. The curves have been smoothed to simulate a receiver bandwidth of $5 \mathrm{MHz}$ and a difference between core and cladding losses of $100 \mathrm{~dB} / \mathrm{km}$ has been assumed for curve (iii). Fig. $2 b$ shows the calculated curves of local attenuation for the 3 cases. It can be seen that for the mechanism involving cladding modes [Fig. $2 a$, curve (iii)], the increase in backscattered power fades back to the normal level for scatter points downstream from the taper as a result of preferential attenuation of power returning in the cladding. The effect is to produce an overshoot in the local attenuation curve [Fig. $2 b$, curve (iii)]. Note that, in practice, mechanisms (ii) and (iii) often coexist.

Experiment: To verify the cladding mode effect, a backscatter attenuation measurement was performed using a semiconductor laser operating at $904 \mathrm{~nm}$ on a graded index fibre which featured a single programmed taper identical to that shown schematically in Fig. 2. Curve 1 of Fig. 3 gives the result for probe-pulse propagation into the contracting taper and clearly shows a marked similarity to curve (iii) of Fig. $2 a$. The

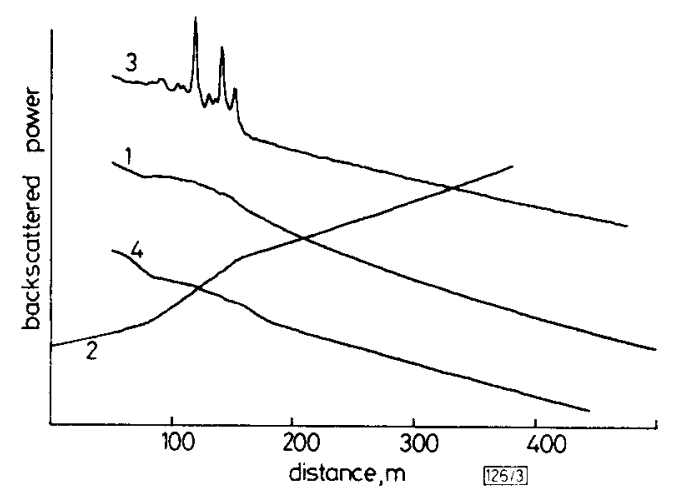

Fig. 3 Measured hackscattered power in silicone-coated graded index fibre with taper dimensions as in Fig. 2

1 Looking into contracting taper

2 Looking into expanding taper

3 As (1) with silicone removed in taper region

4 As (1), index matched

curve returns to the expected backscatter level after a distance of $100 \mathrm{~m}$. indicating a preferential cladding attenuation of $\sim 100 \mathrm{~dB} / \mathrm{km}$, a not unlikely figure for a silicone-clad fibre.
Curve 2 shows the result for propagation in the reverse direction, i.e. into an expanding taper, and is similar to that given in Fig. $2 a$, curve (i). Note, however, that the magnitude of the effects is approximately half that calculated, owing, we believe, to the fact that light scattered from a pulse which only partially excites the guide does not fully excite the guide in the reverse direction.

Further verification of the effect of cladding modes is given in Fig. 3, curve 3, obtained when the silicone coating was removed in the taper region by immersion in concentrated sulphuric acid. A large number of peaks are evident in the trace, presumably as a result of scattering of cladding modes at the fibre/acid interface. These peaks are not evident when viewed from the other end of the fibre (overlaid on curve 2), the result for which remained totally unaffected by coating removal. In order to eliminate the effect of interface scatter, the fibre was immersed in index matching liquid (curve 4). The reduction in local attenuation was then much less apparent: however, it was not possible to remove it completely, an observation which emphasises the persistence of low order cladding modes which contribute to the effect.

Conclusions: The change in backscatter power levels associated with diameter variations in the fibre can be explained in terms of their effect on the reverse-travelling light. A simple model has been developed which indicates that the loss features should be anticorrelated when observed from either end of the fibre, and which gives an upper limit to the magnitude of the effect. Cladding modes have been shown to play a considerable part in the production of negative-going loss features.

Acknowledgments: Acknowledgments are made to $\mathbf{M}$. R. Hadley and R. J. Mansfield for supplying the fibre. The work was supported by UK Ministry of Defence. The authors acknowledge assistance from Pirelli General Cable Co., UK Science Research Council and Australian Telecommunications Commission.

\section{A. J. CONDUIT \\ D. N. PAYNE \\ A. H. HARTOG}

M. P. GOLD

9th March 1981

Department of Electronics

University of Southampton

Southampton SO9 $5 \mathrm{NH}$, England

\section{Reference}

1 CONDUIT, A. J., PAYNE, D. N., and haRTOG, A. H.: 'Optical fibre backscatter-loss signatures: identification of features and correlation with known defects using the two-channel technique'. Sixth European conference on optical communication, York. 1980, pp. $152-154$

$0013-5194 / 81 / 080308-03 \$ 1.50 / 0$ 